\title{
Correction to: Localised heating and intensive magmatic conditions prior to the 22-23 April 2015 Calbuco volcano eruption (Southern Chile)
}

\author{
Eduardo Morgado ${ }^{1,2}$ (D) Daniel J. Morgan ${ }^{1}$ - Jason Harvey ${ }^{1} \cdot$ Miguel-Ángel Parada ${ }^{2,3}$ - Angelo Castruccio ${ }^{2,3}$. \\ Raimundo Brahm ${ }^{2,3,4}$ • Francisco Gutiérrez ${ }^{5}$ - Bogomil Georgiev ${ }^{1}$ • Samantha J. Hammond ${ }^{6}$
}

Published online: 30 December 2019

(C) International Association of Volcanology \& Chemistry of the Earth's Interior 2019

\section{Correction to: Bulletin of Volcanology (2019) 81: 24}

https://doi.org/10.1007/s00445-019-1280-2

In the original version of this article, Table 4 was published incorrectly. The correct Table 4 is given below. This error does not impact the analysis or interpretations of the article.

The online version of the original article can be found at https://doi.org/ $10.1007 / \mathrm{s} 00445-019-1280-2$

Eduardo Morgado eeem@1eeds.ac.uk

1 Institute of Geophysics and Tectonics, School of Earth and Environment, University of Leeds, Leeds LS2 9JT, UK

2 Centro de Excelencia en Geotermia de los Andes (CEGA-FONDAP 15090013), Santiago, Chile

3 Departamento de Geología, Facultad de Ciencias Físicas y Matemáticas, Universidad de Chile, Santiago, Chile

4 Volcanic Risk Solutions, Institute of Agriculture and Environment, Massey University, Palmerston North 4442, New Zealand

5 GeoExpedition, Las Barrancas 25, Pirque, 9480000 Santiago, Chile

6 Environment, Earth \& Ecosystems, The Open University, Milton Keynes MK7 6AA, UK 
Table 4 Whole-rock analyses of samples from the 2015 Calbuco eruption

\begin{tabular}{|c|c|c|c|c|c|c|c|c|}
\hline & Detection limit & $\begin{array}{l}\text { Cal-149A } \\
\text { Lapilli }\end{array}$ & $\begin{array}{l}\text { Cal-149B } \\
\text { Lapilli }\end{array}$ & $\begin{array}{l}\text { Cal-155 } \\
\text { Lapilli }\end{array}$ & $\begin{array}{l}\text { Cal-157 } \\
\text { Bomb }\end{array}$ & $\begin{array}{l}\text { Cal-158 } \\
\text { Bomb }\end{array}$ & $\begin{array}{l}\text { Cal-159 } \\
\text { Bomb }\end{array}$ & $\begin{array}{l}\text { Cal-160 } \\
\text { Bomb }\end{array}$ \\
\hline $\mathrm{SiO}_{2}$ & $0.01(\%)$ & 56.2 & 56.2 & 55.6 & 55.7 & 55.8 & 55.7 & 56.9 \\
\hline $\mathrm{Al}_{2} \mathrm{O}_{3}$ & $0.01(\%)$ & 18.8 & 18.7 & 18.9 & 18.8 & 18.9 & 18.7 & 18.6 \\
\hline $\mathrm{TiO}_{2}$ & $0.01(\%)$ & 0.93 & 0.92 & 0.87 & 0.92 & 0.93 & 0.94 & 0.82 \\
\hline $\mathrm{FeO}$ & $0.2(\%)$ & 4.99 & 4.99 & 5.69 & 5.27 & 5.55 & 5.27 & 5.41 \\
\hline $\mathrm{Fe}_{2} \mathrm{O}_{3}$ & $0.01(\%)$ & 2.87 & 2.77 & 2.22 & 2.6 & 2.41 & 2.71 & 2.17 \\
\hline $\mathrm{MnO}$ & $0.01(\%)$ & 0.16 & 0.16 & 0.17 & 0.17 & 0.17 & 0.15 & 0.16 \\
\hline $\mathrm{MgO}$ & $0.01(\%)$ & 3.93 & 3.9 & 4.27 & 4.14 & 4.2 & 4.25 & 3.81 \\
\hline $\mathrm{CaO}$ & $0.01(\%)$ & 8.04 & 7.96 & 8.21 & 8.16 & 8.14 & 8.12 & 7.63 \\
\hline $\mathrm{Na}_{2} \mathrm{O}$ & $0.01(\%)$ & 3.77 & 3.78 & 3.45 & 3.65 & 3.65 & 3.65 & 3.67 \\
\hline $\mathrm{K}_{2} \mathrm{O}$ & $0.01(\%)$ & 0.62 & 0.64 & 0.53 & 0.61 & 0.61 & 0.61 & 0.67 \\
\hline $\mathrm{P}_{2} \mathrm{O}_{5}$ & $0.01(\%)$ & 0.17 & 0.16 & 0.15 & 0.16 & 0.16 & 0.16 & 0.16 \\
\hline LOI & & -0.3 & -0.3 & -0.2 & -0.3 & -0.3 & -0.3 & -0.1 \\
\hline TOTAL & & 100.48 & 100.18 & 100.06 & 100.18 & 100.52 & 100.26 & 99.99 \\
\hline $\mathrm{Li}$ & $0.1(\mathrm{ppm})$ & 15.1 & 15.7 & 14.9 & 14.8 & 14.2 & 14.7 & 16.9 \\
\hline $\mathrm{Sc}$ & $0.1(\mathrm{ppm})$ & 21.8 & 22.8 & 21.4 & 23.2 & 22.8 & 24 & 19.9 \\
\hline $\mathrm{V}$ & $0.1(\mathrm{ppm})$ & 181.7 & 183.7 & 185.3 & 189.3 & 188.6 & 197.1 & 170.1 \\
\hline $\mathrm{Co}$ & $0.1(\mathrm{ppm})$ & 40.9 & 38.9 & 43.3 & 38.4 & 38.3 & 34.9 & 34.8 \\
\hline $\mathrm{Ni}$ & $0.1(\mathrm{ppm})$ & 10 & 10.8 & 11.7 & 12.9 & 15.7 & 12.9 & 11 \\
\hline $\mathrm{Cu}$ & $0.5(\mathrm{ppm})$ & 44.2 & 45.4 & 24.1 & 50.6 & 47.5 & 46 & 36.9 \\
\hline $\mathrm{Cr}$ & $0.1(\mathrm{ppm})$ & 12.9 & 14.9 & 15.1 & 18.2 & 17.4 & 17 & 15.8 \\
\hline $\mathrm{Zn}$ & 2 (ppm) & 80 & 76 & 79 & 76 & 78 & 81 & 82 \\
\hline $\mathrm{Tl}$ & $0.05(\mathrm{ppm})$ & 0.12 & 0.11 & 0.12 & 0.09 & 0.09 & 0.09 & 0.14 \\
\hline $\mathrm{Rb}$ & $0.1(\mathrm{ppm})$ & 16.5 & 16.4 & 14.3 & 15.9 & 15.2 & 15.8 & 18.4 \\
\hline $\mathrm{Ba}$ & $0.5(\mathrm{ppm})$ & 152.1 & 151.6 & 131.4 & 146 & 139.7 & 145.3 & 164 \\
\hline $\mathrm{Th}$ & $0.1(\mathrm{ppm})$ & 1.1 & 1 & 0.9 & 1 & 1 & 1 & 1.2 \\
\hline $\mathrm{U}$ & $0.1(\mathrm{ppm})$ & 0.3 & 0.3 & 0.3 & 0.3 & 0.3 & 0.3 & 0.4 \\
\hline $\mathrm{Nb}$ & 0.1 (ppm) & 5 & 3.2 & 2.8 & 4.6 & 2.9 & 3.4 & 3.2 \\
\hline $\mathrm{La}$ & $0.1(\mathrm{ppm})$ & 6.4 & 6.4 & 5.6 & 6.2 & 5.9 & 6.1 & 6.9 \\
\hline $\mathrm{Ce}$ & 1 (ppm) & 17 & 17 & 14 & 16 & 16 & 16 & 17 \\
\hline $\mathrm{Pb}$ & $0.1(\mathrm{ppm})$ & 5 & 4.7 & 6.6 & 4 & 4.3 & 4.4 & 5 \\
\hline $\operatorname{Pr}$ & $0.1(\mathrm{ppm})$ & 2.4 & 2.4 & 2 & 2.3 & 2.2 & 2.3 & 2.5 \\
\hline $\mathrm{Sr}$ & 1 (ppm) & 347 & 355 & 375 & 350 & 335 & 352 & 353 \\
\hline $\mathrm{Nd}$ & $0.1(\mathrm{ppm})$ & 11.8 & 11.8 & 9.4 & 11.4 & 10.8 & 11.3 & 11.8 \\
\hline $\mathrm{Zr}$ & $0.1(\mathrm{ppm})$ & 88.5 & 87.3 & 68.3 & 83.8 & 79.6 & 84 & 89.8 \\
\hline $\mathrm{Sm}$ & $0.1(\mathrm{ppm})$ & 3.3 & 3.3 & 2.5 & 3.1 & 3 & 3.2 & 3.1 \\
\hline $\mathrm{Eu}$ & $0.1(\mathrm{ppm})$ & 1 & 1.1 & 1 & 1 & 1 & 1 & 1 \\
\hline $\mathrm{Gd}$ & $0.1(\mathrm{ppm})$ & 3.5 & 3.5 & 2.6 & 3.4 & 3.2 & 3.4 & 3.3 \\
\hline $\mathrm{Tb}$ & $0.1(\mathrm{ppm})$ & 0.6 & 0.6 & 0.4 & 0.6 & 0.5 & 0.6 & 0.5 \\
\hline Dy & 0.1 (ppm) & 3.6 & 3.6 & 2.6 & 3.4 & 3.3 & 3.5 & 3.3 \\
\hline Но & $0.1(\mathrm{ppm})$ & 0.7 & 0.8 & 0.6 & 0.8 & 0.7 & 0.8 & 0.7 \\
\hline $\mathrm{Er}$ & $0.1(\mathrm{ppm})$ & 2.2 & 2.2 & 1.7 & 2.1 & 2 & 2.1 & 2 \\
\hline $\mathrm{Y}$ & $0.1(\mathrm{ppm})$ & 22.5 & 23.2 & 17.1 & 22.1 & 20.9 & 22.2 & 21.3 \\
\hline $\mathrm{Yb}$ & $0.1(\mathrm{ppm})$ & 2.2 & 2.3 & 1.7 & 2.2 & 2.1 & 2.1 & 2 \\
\hline $\mathrm{Lu}$ & $0.05(\mathrm{ppm})$ & 0.33 & 0.34 & 0.25 & 0.32 & 0.31 & 0.33 & 0.32 \\
\hline
\end{tabular}

